Z. klin. Chem. u. klin. Biochem.

8. Jg., S. 409-412, Juli 1970

\title{
Die therapeutische Beeinflussung einer experimentellen Nephritis durch homologe Nierenmitochondrien ${ }^{1}$ )
}

Von H. SARRE und E. Vonend

Medizinische Poliklinik der Universität Freiburg im Breisgau

(Eingegangen am 9. Februar 1970)

Ratten, in denen durch Anti-Nieren-Serum eine „Masugi“-Nephritis erzeugt worden war, wurden mit verschiedenen Zellpartikelfraktionen intravenös bezw. intraperitoneal behandelt. Als quantitative Erfolgskriterien dienten vor allem die Verminderung der Proteinurie und des Serum-Reststickstoffs sowie die Normalisierung des Serumproteins. Günstige Wirkungen wurden nach intravenöser Applikation intakter, homologer Nierenmitochondrien, aber auch mit ultraschallbehandelten Mitochondriendispersionen erzielt. Die anderen Fraktionen waren bei intravenöser Injektion immunologisch unverträglich, bei intraperitonealer Applikation gänzlich oder nahezu wirkungslos.

\section{The therapeutic effect of bomologous kidney mitocbondria in experimental nepbritis}

„Masugi“"-nephritis was produced in rats by treatment with antikidney serum. The animals were then treated with various cell particle fractions by intravenous and intraperitoneal injection respectively. The therapeutic effects were quantitized by measurements of proteinuria, and the non-proteinnitrogen and total protein of serum. Favourable results were obtained by intact homologous kidney mitochondria as well as by mitochondrial sonicates applied intravenously The other particulate cell fractions, when injected intravenously, showed immunologic intolerance and were rather ineffective when applied intraperitoneally.

Die durch heterologe nephrotrope Antikörper erzeugte Glomerulo-Nephritis-Nephrose („Masugi-Nephritis“) entspricht sowohl klinisch als auch histologisch weitgehend der menschlichen Glomerulonephritis (vgl. FAHR (1)) und stellt deshalb ein gutes tierexperimentelles Modell für pathogenetische, funktionelle und therapeutische Studien dar. Anknüpfend an Ergebnisse von Hötzl, LAudArn und Lüders (2-10), die durch Behandlung mit homologen Lebermitochondrien eine klinische und histologische Besserung der durch Tetrachlorkohlenstoff erzeugten Leberschädigung sahen, wurde versucht, „Masugi-“Nephritiden mit homologen Nierenmitochondrien $\mathrm{zu}$ behandeln. SCHNELIBACHER (11) und Köpp (12) aus unserem Arbeitskreis hatten hierbei bereits positive Ergebnisse erzielt. In dieser Arbeit werden diese erweitert und auf andere Zellfraktionen sowie auf Ultraschall behandelnde Mitochondrien ausgedehnt.

\section{Material und Methoden}

In allen Versuchen wurden männliche Inzuchtratten (WISTAR, $100-200 \mathrm{~g}$ ) verwandt, die in Stoffwechselkäfigen gehalten und täglich mit einer definierten Menge Standarddiät (Firma LATZ, Euskirchen), zur Vermeịdung diätabhängiger Proteinurieschwankungen (13), gefüttert wurden.

Anti-Rattennieren-Serum wurde aus Kaninchen nach der Methode von Rother (14) gewonnen. 0,4-0,5 ml Anti-RattennierenSerum $/ 100 \mathrm{~g}$ Körpergewicht führten zu einer deutlichen Nephritis. Die Aktivität des Antiserums sank auch in tiefgefrorenem $\mathrm{Zu}-$ stand in einem Zeitraum von einigen Monaten allmählich ab und mußte vor Wiederverwendung neu bestimmt werden.

Protein wurde nach der Biuret-Methode nach 1 c. (15), Rest-

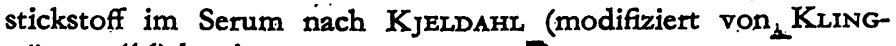
MÜLLER (16) bestimmt.

Die Zellfraktionen wurden aus den dekapsulierten Nieren frisch getöteter Ratten gewonnen: die zerkleinerten Organe wurden

1) Mit Förderung durch die Deutsche Forschungsgemeinschaft. in 0,25 M Saccharose-Lösung (0,25 M Tris-HCl-Puffer $\mathrm{pH} \mathrm{7,4} \mathrm{ent-}$ haltend) nach PotTer und ElvehJEM (17) homogenisiert und dann nach der Mcthode von SchNeIDer und Hogeboom (18) in einer Sorvall-RC2-B-Zentrifuge fraktioniert. Die sahniggelbe Auflagerung des bei $700 \mathrm{~g}$ (10 Min.) sedimentierten und mit gleichem Medium gewaschenen Niederschlags wird im folgenden als ,,Zelltrümmerfraktion" bezeichnet; elektronenoptisch ließen sich darin neben einigen Mitochondrien Kernfragmente und Faserlemente erkennen. Die bei $5000 \mathrm{~g}$ ( $10 \mathrm{Min}$.) sedimentierte und $2 \mathrm{mal}$ bei $24000 \mathrm{~g}$ (19 Min.) rezentrifugierte Fraktion besteht aus elektronenmikroskopisch fast reinen Mitochondrien (,Mitochondrienfrak-

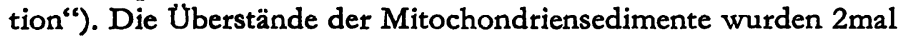
je $1 \mathrm{Std}$. bei $41000 \mathrm{~g}$ zentrifugiert; das Sediment enthielt neben Mikrosomen vorwiegend Ergastoplasma-Elemente und Zellmembranen (,Membranfraktion"). Alle partikulären Fraktionen wurden im genannten Medium im Verhältnis 1:8 suspendiert und umgehend in die Tierversuche eingesetzt.

Ein Teil der frischbereiteten Mitochondriensuspension wurde insgesamt $10 \mathrm{Min}$. lang unter Wasserkühlung ultrabeschallt ${ }^{1}$ ), wobei die Beschallung jeweils nach 30 Sek. für 30 Sek. unterbrochen wurde. Es resultierte eine klare, braune Dispersion.

Die Mitochondriensuspensionen wurden nach der Methode von Bernath und Singer (19) nach ihrem Gehalt an Succinat-Dehydrogenase und an Protein standartisiert. Die Bestimmung von Succinat-Dehydrogenase erfolgte im Warburg-Apparat mit Phenazinmethosulfat. Die Einstellung der Mitochondriensuspensionen entspricht der von SchINEL L BACHER(11), Köpp(12) und KuHL (24). Diese Autoren geben als Aktivitätseinheit für Succinat-Dehydrogenase das Volumen an Mitochondriensuspension an, das $1 \mu \mathrm{l} \mathrm{O}_{2} / \mathrm{Min}$. veratmet. Bezogen auf $\mathrm{mg}$ Protein ergibt sich daraus die spezifische Aktivität, die durchschnittlich bei 7-14 lag. Wie SchNellBACHER und Köpp (12) nachweisen konnten, liegt die Dosis für den besten therapeutischen Effekt der Mitochondriensuspension auf die experimentelle Rattennephritis bei 2,5-5,0 Aktivitätseinheiten entsprechend $0,36 \mathrm{mg}$ Protein. Wir verdünnten die Mitochondriensuspensionen mit isotoner Saccharoselösung so, $\mathrm{da}$ die angegebenen Aktivitätseinheiten in $0,2 \mathrm{ml}$ Lösung enthalten waren. Die Lösung hatte dann einen Proteingehalt von $1,8 \mathrm{mg} / \mathrm{ml}$ und enthielt 2,5-5,0 Aktivitätseinheiten SuccinatDehydrogenase pro $0,2 \mathrm{ml}$. Sadayoshr, Hashimoto und Mitarbeiter (26) führten ähnliche Versuche mit Mitochondrien-

1) Schoeller \& Co., Frankfurt, 20 kH, 750 W, 0,6 A Betriebsstärke. 
suspensionen durch, deten Proteingehalt $1,5 \mathrm{mg} / \mathrm{ml}$ betrug. $\mathrm{Da}$ die Succinat-Dehydrogenasc-Aktivitätsmessungen sehr aufwendig sind, standardisierten wir die Suspensionen später nach dem Eiweißgehalt, der auch dabei $1,8 \mathrm{mg} / \mathrm{ml}$ betrug. Die übrigen Zellfraktionen wurden auf den gleichen Eiweißgehalt eingestellt. Daneben führten wir Aktivitätsmessungen der Cytochrom-cOxydase nach DaLLNER und Mitarbeiter (20) durch, um sicherzustellen, daß wir intakte Mitochondrien verwendeten. Die Messungen erfolgten jedoch wegen der Trübung der Mitochondriensuspensionen nur qualitativ.

Die Signifikanzberechnungen wurden nach FISCHER-STUdeNT mit Hilfe der Geigy-Tabellen durchgeführt.

\section{Ergebnisse}

Die Wirkung verschiedener Zellfraktionen auf „Masugi"-nephritische Ratten wurde in zwei Versuchsreihen geprüft. Als quantitative Parameter eines Therapieerfolges wurden herangezogen: die wöchentliche Gewichtsdifferenz der Tiere, die tägliche Urinmenge und der Urinproteingehalt und (am Ende des Versuchs) der Reststickstoff im Serum und das Gesamtprotein des Serums. Diese Werte wurden verglichen mit denen von Kontrolltieren, deren gleichzeitig erzeugte „Masugi“Nephritis unbehandelt blieb.

In einer ersten Versuchsreihe wurde die Wirkung intraperitoneal applizierter „Membranfraktionen" und ,Zelltrümmerfraktionen" sowie intravenös injizierter Mitochondrien untersucht. 72 Ratten wurde je $0,4 \mathrm{ml}$ AntiRattennieren-Serum $/ 100 \mathrm{~g}$ Tier. intravenös in die Schwanzrene injiziert. Dies führte bei allen Tieren $z u$ einer mittelschweren Nephritis-Nephrose, die bei den Kontrollen ihren Höhepunkt am 8. Tag mit einer mittleren Proteinurie von $168 \mathrm{mg} / \mathrm{Tag}$ erreichte. Die Tiere wurden in 4 gleiche Kollektive aufgeteilt, deren eines als Kontrolle diente; einem zweiten wurde die Mitochondrien-Suspension (5 Aktivitätseinheiten SuccinatDehydrogenase) in die Schwanzvene injiziert, die dritte und vierte Gruppe erhielt jeweils die gleiche Menge (auf Proteingehalt bezogen) an "Zelltrümmer"- bzw. "Zellmembran"-Fraktion intraperitoneal. Versuche an Normalratten hatten ergeben $(11,12)$, daß die intravenöse Applikation von Nierenmitochondrien von den Tieren gut vertragen wurden, also weder eine toxische noch eine immunologische Reaktion zu befürchten ist. Dagegen führten die beiden anderen Zellfraktionen zu schweren Schockzuständen und zum Tod der Tiere, so daß hier nur die intraperitoneale Verabfolgung möglich war. Aus der Abbildung 1 sowie den Tabellen 1 und 2 ist zu ersehen, daß die Behandlung mit Mitochondrien-Sus- pension zu einer signifikanten Erniedrigung der Proteinurie, zu einer Normalisierung der Serumproteinwerte und $\mathrm{zu}$ einer deutlichen Verringerung des Serum-Reststickstoffes gegenüber der Kontrollgruppe führte. Auch hinsichtlich der äußeren Symptome, wie Ödembildung,

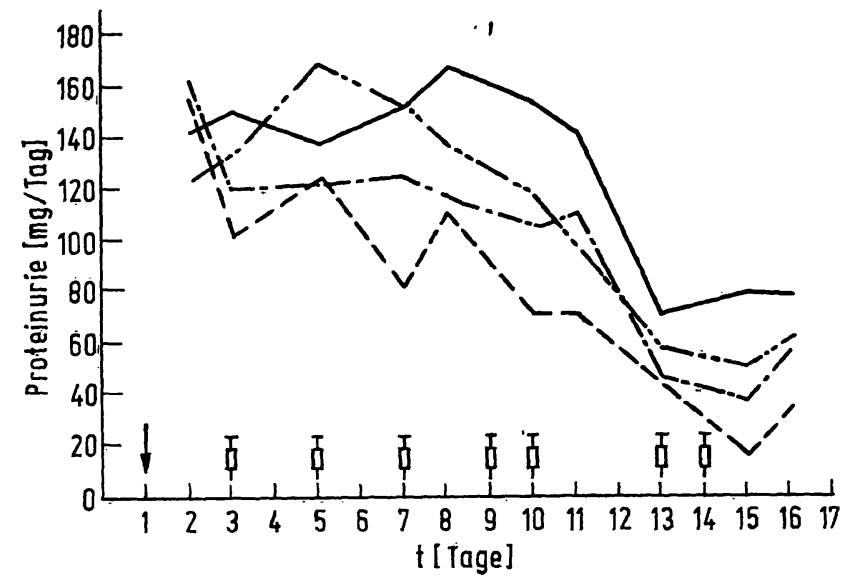

Abb. 1

Mittlere Proteinurie von vier Gruppen von Ratten mit "Masugi-“ Nephritis (je 17-19 Tiere), I. Hauptserie. Die mit Injektionsspritzen jeweils $0,4 \mathrm{ml}$ Anti-Rattennieren-Serum am 1. Tag (Pfeil) Behandlung: ohne:

Zellmembran-Fraktion i.p. $-\cdots-\cdots$
Zelltrümmer-Fraktion i. p. $-\cdots-$ Mitochondrien-Fraktion i. v. - -

Tab. 1

Ergebnisse der Signifikanz-Berechnungen

I. Versuchsserie

1. Mitochondrien-Gruppe-Kontrollgruppe

Proteinurie/Tag

5. Tag P: $0,60-0,50$

11. Tag P: $0,01-0,00$

15. Tag P: $0,01-0,001$

P: $\quad 0,001$

Gesamt-Eiweiß

im Serum

P: $\quad 0,001$

cht signifikant signifikant signifikant hochsignifikant

Rest-Stickstoff

hochsignifikant

2. Zelltrümmer-Gruppe-Kontrollgruppe

Proteinurie/Tag

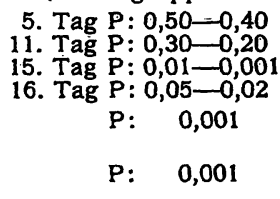

nicht signifikant nicht signifikant signifikan signifikant hochsignifikant

Gesamt-Eiweiß im Serum Rest-Stickstoff im Serum P: $\quad 0,001$ hochsignifikant

3. Zellmembranen-Gruppe-Kontrollgruppe

Proteinurie/Tag

5. Tag P: $0,20-0,10$

11. Tag P: $0,10-0,05$

15. Tag P: $0,20-0,10$

Gesamt-Eiweiß

P: $0,01-0,001$

im Serum

Rest-Stickstoff $P: 0,01-0,00$

P: $\quad 0,001$

nicht signifikant nicht signifikant nicht signifikant hochsignifikant

im Serum

Tab. 2

Ergebnis der I. Versuchsserie

\begin{tabular}{|c|c|c|c|c|c|c|c|c|c|}
\hline Gruppe & Applikation & $\begin{array}{l}\text { Sterb- } \\
\text { lich- } \\
\text { keit }\end{array}$ & \multicolumn{2}{|c|}{$\begin{array}{l}\text { Körpergewicht, } \\
\text { Mittelwerte }\end{array}$} & $\begin{array}{c}\text { verringerte } \\
\text { Proteinurie } \\
P \\
\text { signifikant }\end{array}$ & \multicolumn{2}{|c|}{$\begin{array}{c}\text { Gesamteiweiß } \\
\text { im Serum } \\
\text { Mittelwerte } \\
P\end{array}$} & \multicolumn{2}{|c|}{$\begin{array}{l}\text { Rest-Stickstoff } \\
\text { im Serum, } \\
\text { Mittelwerte }\end{array}$} \\
\hline $\begin{array}{l}\text { Mitochondrien- } \\
\text { Tiere }(n=18)\end{array}$ & $\begin{array}{l}\text { intra- } \\
\text { venős }\end{array}$ & 2 Tiere & $133 \mathrm{~g}$ & $132 \mathrm{~g}$ & ab 11. Tag & $6,5 \mathrm{~g} / 1.00 \mathrm{ml}$ & 0,001 & $44,5 \mathrm{mg} / 100 \mathrm{ml}$ & 0,001 \\
\hline $\begin{array}{l}\text { Zelltrümmer- } \\
\text { Tiere }(n=18)\end{array}$ & $\begin{array}{l}\text { intraperi- } \\
\text { toneal }\end{array}$ & 1 Tier & $126 \mathrm{~g}$ & $131 \mathrm{~g}$ & ab 15. Tag & $6,1 \mathrm{~g} / 1.00 \mathrm{ml}$ & 0,001 & $43,7 \mathrm{mg} / 100 \mathrm{ml}$ & 0,001 \\
\hline $\begin{array}{l}\text { Zellmembranen- } \\
\text { Tiere }(n=17)\end{array}$ & $\begin{array}{l}\text { intraperi- } \\
\text { toneal }\end{array}$ & 1 Tier & $132 \mathrm{~g}$ & $130 \mathrm{~g}$ & $\begin{array}{l}\text { nicht } \\
\text { signifikant }\end{array}$ & $5,1 \mathrm{~g} / 100 \mathrm{ml}$ & $0,01-0$, & $50,0 \mathrm{mg} / 100 \mathrm{ml}$ & 0,001 \\
\hline $\begin{array}{l}\text { Kontroll- } \\
\text { Tiere }(n=19)\end{array}$ & - & 2 Tiere & $126 \mathrm{~g}$ & $124 \mathrm{~g}$ & - & $4,5 \mathrm{~g} / 100 \mathrm{ml}$ & - & $63,7 \mathrm{mg} / 100 \mathrm{ml}$ & - \\
\hline
\end{tabular}


Fellbeschaffenheit und Freßlust, zeigten die behandelten Tiere einen deutlich verbesserten Zustand.

Die mit der "Zelltrümmerfraktion" behandelten Nephritis-Tiere zeigten nur hinsichtlich der Serumwerte und des äußeren Eindrucks eine Verbesserung, die Verringerung der mittleren Proteinurie war kaum signifikant.

Der Effekt der „Membranfraktion“" war wesentlich geringer, sowohl in bezug auf das Serumprotein als auch auf den Reststickstoff und den äußeren Eindruck.

Die 2. Versuchsserie (Abb. 2, Tab. 3 und 4) umfaßte 73 Tiere, die jeweils $0,5 \mathrm{~m} l$ Anti-Rattennieren-Serum/ $100 \mathrm{~g}$ Tiergewicht einer weniger aktiven Charge AntiRattennieren-Serum intravenös erhielten; sie zeigten

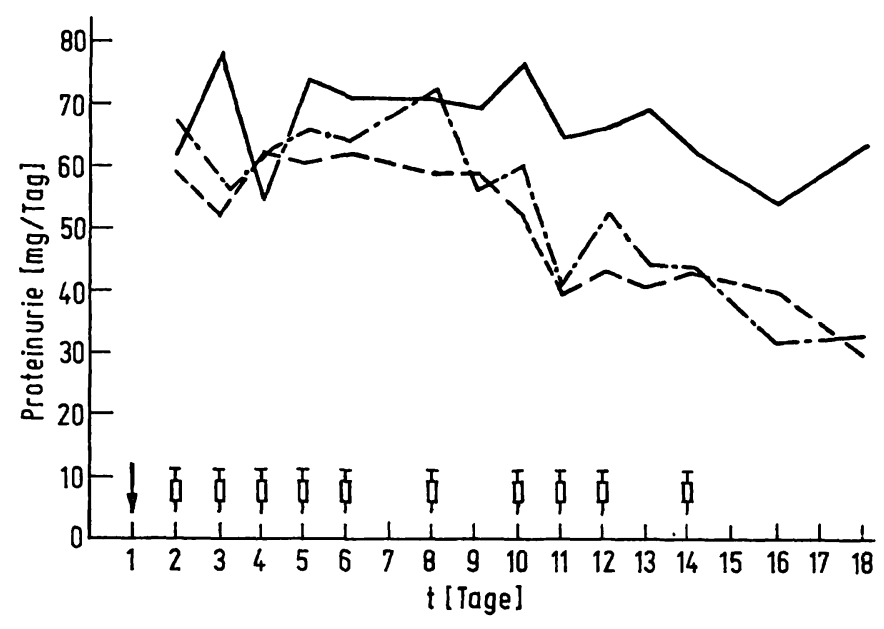

Abb. 2

Mittlere Proteinurie von drei Gruppen von Ratten mit "Masugi“Nephritis (je 24 bis 25 Tiere), II. Hauptserie. Die mit Injektionsspritzen markierten Tage geben die Behandlungstage an. Injiziert wurden jeweils $0,5 \mathrm{ml}$ Anti-Rattennieren-Serum am 1. Tag.

Behandlung: ohne

Mitochondrienfraktion i. v. . . -

Mitochondrienfraktion, ultrabeschallt i. v. - . .

Tab. 3

Ergebnisse der Signifikanz-Berechnungen

\begin{tabular}{|c|c|c|}
\hline \multicolumn{3}{|c|}{$\begin{array}{l}\text { II. Versuchsserie } \\
- \text { Kontrollgruppe }\end{array}$} \\
\hline Proteinurie/Tag & $\begin{array}{l}\text { 6. Tag } P: 0,50-0,40 \\
\text { 11. Tag P: } 0,001 \\
\text { 16. Tag } P: 0,05-0,02\end{array}$ & $\begin{array}{r}\text { nicht signifikant } \\
\text { hochsignifikant } \\
\text { signifikant }\end{array}$ \\
\hline $\begin{array}{l}\text { Gesamt-Eiweiß } \\
\text { im Serum }\end{array}$ & $P: \quad 0,001$ & hochsignifikant \\
\hline $\begin{array}{l}\text { Rest-Stickstoff } \\
\text { im Serum }\end{array}$ & $P: 0,10-0,05$. & nicht signifikant \\
\hline \multicolumn{3}{|c|}{ 2. Mitochondrien-Gruppe-ultrabeschallt-Kontrollgruppe } \\
\hline Proteinurie/Tag & $\begin{array}{l}\text { 6. Tag P: } 0,70-0,60 \\
\text { 11. Tag } P: 0,01-0,001 \\
\text { 16. Tag } P: \quad 0,001\end{array}$ & $\begin{array}{r}\text { nicht signifikant } \\
\text { signifikant } \\
\text { hochsignifikant }\end{array}$ \\
\hline $\begin{array}{l}\text { Gesamt-Eiweiß } \\
\text { im Serum }\end{array}$ & . P: 0,001 & hochsignifikant \\
\hline $\begin{array}{l}\text { Rest-Stickstoff } \\
\text { im Serum }\end{array}$ & $P: 0,90-0,80$ & nicht signifikant \\
\hline
\end{tabular}

durchweg eine leichtere Verlaufsform der Nephritis (Proteinurie am 3. Tag: 78,5 mg Protein/Tag). 25 Tiere dienten als Kontrollgruppe; 24 Ratten wurde intravenös eine Mitochondrien-Suspension (5 Aktivitätseinheiten Succinat-Dehydrogenase), weiteren 24 Tieren in gleicher Weise eine ultrabeschallte Mitochondrien-Suspension gleichen Proteingehalts injiziert. In beiden Versuchsgruppen fiel die Proteinurie erst in der zweiten Behandlungswoche signifikant gegenüber den Kontrollen ab. Die Serumproteinwerte am Ende des Versuchs waren gegenüber den Kontrollen erhöht und im Bereich der Norm. Die unbehandelten Nephritis-Tiere zeigten hinsichtlich ihres Körpergewichts, ihres äußeren Zustands und ihrer Mortalität einen deutlich schlechteren $\mathrm{Zu}$ stand als die behandelten Tiere.

\section{Diskussion}

Der Therapieerfolg an der „Masugi“-Nephritis der Ratte mit homologen Nierenmitochondrien wurde durch quantifizierbare klinisch-chemische Untersuchungen bestimmt und seine Signifikanz gegenüber unbehandelten Masugi-Nephritis-Tieren festgelegt. Hinsichtlich aller der für eine Nephritis charakteristischen Parameter erwies sich die Behandlung mit einer MitochondrienSuspension als erfolgversprechend. Erstaunlicherweise tritt der gleiche Effekt auch ein, wenn anstelle intakter Nierenmitochondrien ultrabeschallte Mitochondrienpräparate verwendet werden. Die strukturelle oder funktionelle Integrität dieser Zellpartikeln scheint demnach für die therapeutische Wirkung nicht entscheidend $\mathrm{zu}$ sein; damit verliert auch eine geäußerte Vermutung (9), daß geschädigte Mitochondrien in den Leberzellen durch die Mitochondrieninfusion ersetzt werden könnten, an Wahrscheinlichkeit. Es ist weiteren Untersuchungen vorbehalten, $z u$ entscheiden, ob der therapeutische Effekt auf submitochondriale Partikel oder molekulare Komponenten der Mitochondrien zurückzuführen ist.

Die Untersuchungen von HötZL, LAUDAHN und LüDERS (2-10) hatten gezeigt, daß durch die intravenöse Applikation von homologen Mitochondrien die Wiederherstellung einer Tetrachlorkohlenstoff-geschädigten Leber beschleunigt werden kann. Aus diesen Versuchen konnte weiterhin entnommen werden (7), daß eine gewisse Organaffinität $z$ wischen den applizierten Mitochondrien und ihrem Muttergewebe besteht und die Organaffinität verstärkt wird, wenn eine Schädigung des korrespondierenden Gewebes vorhanden ist. Leistungsverbesse-

Tab. 4

Ergebnisse der II. Versuchsserie

\begin{tabular}{|c|c|c|c|c|c|c|c|c|c|c|}
\hline \multirow{2}{*}{$\begin{array}{l}\text { Gruppe } \\
\begin{array}{l}\text { Mitochondrien- } \\
\text { Tiere }(\mathrm{n}=24)\end{array}\end{array}$} & \multirow{2}{*}{$\begin{array}{c}\text { Applikation } \\
\begin{array}{l}\text { intra- } \\
\text { venös }\end{array}\end{array}$} & \multirow{2}{*}{$\begin{array}{l}\begin{array}{c}\text { Sterb- } \\
\text { lich- } \\
\text { keit }\end{array} \\
3 \text { Tiere }\end{array}$} & \multicolumn{2}{|c|}{$\begin{array}{c}\text { Körpergewicht } \\
\text { Mittelwerte } \\
\text { zu Beginn am Ende }\end{array}$} & $\begin{array}{c}\text { verringerte } \\
\text { Proteinurie } \\
P \\
\text { signifikant }\end{array}$ & \multicolumn{2}{|c|}{$\begin{array}{c}\text { Gesamteiweiß } \\
\text { im Serum } \\
\text { Mittelwerte } \\
\end{array}$} & \multicolumn{3}{|c|}{$\begin{array}{l}\text { Rest-Stickstoff } \\
\text { im Serum } \\
\text { Mittelwerte }\end{array}$} \\
\hline & & & $152 \mathrm{~g}$ & $161 \mathrm{~g}$ & ab 11. Tag & $6,1 \mathrm{~g} / 100 \mathrm{ml}$ & 0,001 & $40,4 \mathrm{mg} / 100 \mathrm{ml}$ & nicht & t signif. \\
\hline $\begin{array}{l}\text { Mitochondrien- } \\
\text { Tiere }(n=24) \\
\text { (ultrabeschallt) }\end{array}$ & $\begin{array}{l}\text { intra- } \\
\text { venös }\end{array}$ & kein Tier & $148 \mathrm{~g}$ & $152 \mathrm{~g}$ & ab 11. Tag & $6,3 \mathrm{~g} / 100 \mathrm{ml}$ & 0,001 & $34,3 \mathrm{mg} / 100 \mathrm{ml}$ & nicht & t signif. \\
\hline $\begin{array}{l}\text { Kontroll- } \\
\text { Tiere }(n=25)\end{array}$ & - & 6 Tiere & $148 \mathrm{~g}$ & $123 \mathrm{~g}$ & - & $4,7 \mathrm{~g} / 100 \mathrm{ml}$ & - & $34,7 \mathrm{mg} / 100 \mathrm{ml}$ & & 一 \\
\hline
\end{tabular}


rungen geschädigter Herzmuskeln waren bei der Behandlung mit Herzmuskelmitochondrien beobachtet worden (21-23). Andererseits erwiesen sich, nach Befunden unseres Arbeitskreises (11, 12, 24), intraperitoneal injizierte Lebermitochondrien als wirkungslos bei der „Masugi"-Nephritis. Versuche von KuHz (24) sprechen dafür, daß jedoch auch heterologe Nierenmitochondrien einen günstigen Effekt haben.

Ebenso, wie auch von LAUDAHN und LüDERS hinsichtlich der Lebermitochondrien beschrieben, spielt die Dosierung bei diesen Therapieversuchen eine wesentliche Rolle. Eine optimale Mitochondrien-Dosis, entsprechend 2,5-5 Succinat-Dehydrogenase-Einheiten, war von SCHNELlBaCHer (11) und Köpp (12) ermittelt worden; niedrigere hatten entsprechend geringere, höhere jedoch bereits schädliche Wirkungen auf die Nephritis-Tiere.
Ein entscheidendes Problem bei der therapeutischen i. v. Applikation von Zellpartikeln ist die immunologische Verträglichkeit. Während sowohl die bei $750 \mathrm{~g}$ sedimentierte als auch die "Membranfraktion" zu starken anaphylaktischen Prozessen führen, sind Mitochondrien auch intravasal sehr gut verträglich (25). Die Tatsache, daß sowohl die "Zelltrümmerfraktion" als auch die "Membranfraktion" bei intraperitonealer Applikation relativ gut vertragen werden, erhebt die Frage, wie rasch und in welchem Zustand diese Partikel in die allgemeine Zirkulation resorbiert werden; insofern ist ein Vergleich mit der Wirkung i. v. applizierter Mitochondrien-Suspensionen nicht ohne weiteres möglich.

Herrin Dr. P. Rołrk, jetzt Pathologisches Institut der Universität Basel, danken wir für die Durchführung der elektronen-optischen Kontrollen, der Deutschen Forschungsgemeinschaft für die Unterstützung dieser Arbeit.

\section{Literatur}

1. FAHR, Th., Klin. Wschr., 15, 505 (1936). - 2. HötzL, H. A. und G. LaUdahn, Ärztl. Wschr. 11, 634 (1956). - 3. LaUdahN, G., Ärztl. Forsch. 10, 513 (1956). - 4. Laudahn, G. und C. J LüDERS, I. Nitteilung; Virchow's Arch. 329, 581 (1957). 5. H. A. HötzL, G. LAUdAHN und C. J. LüDERS, Berlin Med Hefte 10, 187 (1960). - 6. HötzL, H. A., Münch. med. Wschr. 102, 1670 (1960). - 7. Laudahn, G., C. J. Lüders und W. SCHUMACHER, Zbl. exper. Med. 133, 328 (1960). - 8. LAUdAHN, G. und C. J. LüDERs, II. Mitteilung; Arzneimittel-Forsch., Aulendorf 10, 781 (960). - 9. Laudahn, G. und C. J. Lüders, ArzneimittelForsch., Aulendorf 10, 978 (1960). - 10. HörZL, H. A., Therap.woche, Karlsruhe 11, 506 (1961). - 11. Schnellbacher, E., Inaugural-Diss., Freiburg i. Br., (1966). - 12. Köpp, P.: InauguralDiss., Freiburg i. Br., (1967). - 13. SMADEL, J. E. und L. E. FARR, J. Exper. Med. 70, 615 (1939). - 14. RothER, K., „Experimentelle Nierenkrankheiten" in: Handbuch d. Exper. Pharm. New Series. Herausg. O. Eichler, A. Farah, H. Herken und A.
WeLch., Vol. XVỊ, Teil 4: Niere, Nierenbecken, Blase. SpringerVerl. Heidelberg 1965, p. 1. - 15. Frank, H. und Ph. Köcher, Dtsch. Atch. Klin. Med. 197, 181 (1950). - 16. KIINGmÜLLER, Z. analyt. Chem. 131, 17 (1950). - 17. Portrer, V. R., E. A. Elvenjem, J. biol. Chemistry 114, 495 (1936). - 18. SChNeider, W. C. und G. H. Hogeboom, J. biol. Chemistry 183, 123 (1949). 19. Bernath P. und T. P. Singer, Methods in Enzymol. 5, 597 (1962). - 20. Dallner, G., P. Stekevitz und G. Ë. Palade, J. Cell Biol. 30, 97 (1966). - 21. Nüssgen, W. und H. Stobor, Berl. Med. 7, 364 (1956). - 22. SAuErTeIG, E., Im Sitzungsbericht d. Berliner Pathologen-Vereinig. v. 25. 6. 1957; Zbl. allg. Path. 97, 205 (1957/58). - 23. ReIsky, P., Berl. Med. 11, 170 (1960). - 24. KuHu, U., Inaugural-Diss., Freiburg i. Br. (1970). 25. Henle, W., L. A. Chambers und V. Groupe, J. Exper. Med. 74, 495 (1914). - 26. Sadayoshi Hashimoto, S. Komatsu und R. A. Cowley. Amer. Surgeon 32, 231 (1966).
Prof. Dr. H. Sarre Med. Poliklinik 78 Freiburg/i. Br. Hermann-Herder-Str. 6 